\title{
Two cases of Antley-Bixler syndrome caused by mutations in different genes, FGFR2 and POR
}

\author{
Hyewon Woo, Jung Min Ko ${ }^{1,2 *}$, Choong Ho Shin', and Sei Won Yang ${ }^{1}$ \\ ${ }^{1}$ Department of Pediatrics, Seoul National University Children's Hospital, Seoul National University College of Medicine, Seoul, Korea \\ ${ }^{2}$ Research Coordination Center for Rare Diseases, Seoul National University Hospital, Seoul, Korea
}

Antley-Bixler syndrome (ABS) is a rare form of syndromic craniosynostosis with additional systemic synostosis, including radiohumeral or radioulnar synostosis. Another characteristic feature of $A B S$ is mid-facial hypoplasia that leads to airway narrowing after birth. ABS is associated with mutations in the FGFR2 and POR genes. Patients with POR mutations present with either skeletal manifestations or congenital adrenal hyperplasia with ambiguous genitalia. We report here two cases of $A B S$ caused by mutations in FGFR2 and POR. Although the patients had craniosynostosis and radiohumeral synostosis in common and cranioplasty was performed in both cases, the male with $P O R$ mutations showed an elevated level of $17 \alpha$-hydroxyprogesterone during newborn screening and was diagnosed with congenital adrenal hyperplasia by adrenocorticotropic hormone stimulation. This patient has been treated with hydrocortisone and fludrocortisone. He had no ambiguous genitalia but had bilateral cryptorchidism. On the other hand, the female with the FGFR2 mutation showed severe clinical manifestations: upper airway narrowing leading to tracheostomy, kyphosis of the cervical spine, and coccyx deformity. ABS shows locus heterogeneity, and mutations in two different genes can cause similar craniofacial and skeletal phenotypes. Because the long-term outcomes and inheritance patterns of the disease differ markedly, depending on the causative mutation, early molecular genetic testing is helpful.

Key words: Antley-Bixler syndrome phenotype, Craniosynostoses, Congenital adrenal hyperplasia, FGFR2, POR.

\section{Introduction}

Craniosynostosis is defined as partial or complete premature fusion of skull sutures. The incidence of craniosynostosis is $\sim 1: 2,500$ [1]. Syndromic craniosynostosis are estimated to comprise $15 \%$ of all cases and over 180 craniosynostosis syndromes have been identified [2]. Mutations in the fibroblast growth factor receptor 2 gene (FGFR2) are associated with various craniosynostosis syndromes, including Apert, Crouzon, Pfeiffer, and Antley-Bixler syndromes (ABS; OMIM \#207410,
\#201750). FGFR2 mutations show variable clinical penetrance and patients with the same FGFR2 mutation can exhibit diverse clinical features. Therefore, FGFR2-related craniosynostosis syndromes are usually named according to the accompanying extra-cranial manifestations.

$A B S$ is a rare type of craniosynostosis syndrome. $A B S$ is typically distinguished by systemic bony fusions of skull sutures and other joints. Skeletal manifestations include radiohumeral or radioulnar synostosis, arachnodactyly, multiple joint contractures, and bowing of the femora. Mid-face hypoplasia

Received: 20 November 2015, Revised: 4 January 2016, Accepted: 10 January 2016, Published: 30 June 2016

${ }^{*}$ Corresponding author: Jung Min Ko, M.D., Ph.D.

Department of Pediatrics, Seoul National University College of Medicine, Seoul National University Children's Hospital, 101 Daehak-ro, Jongno-gu, Seoul 03080, Korea.

Tel: +82-2-2072-3570, Fax: +82-2-743-3455, E-mail: jmko@snu.ac.kr

Conflict of interest: The authors declare that they do not have any conflicts of interest.

(c) This is an open-access article distributed under the terms of the Creative Commons Attribution Non-Commercial License (http://creativecommons.org/licenses/by-nc/4.0/) which permits unrestricted non-commercial use, distribution, and reproduction in any medium, provided the original work is properly cited.

(c) Copyright 2016 by the Korean Society of Medical Genetics 
leading to proptosis and airway narrowing is also present in most patients. In 2004, the second causative gene for ABS, POR, was identified [3]. POR mutations are inherited in an autosomal recessive fashion, while FGFR2 mutations are inherited in an autosomal dominant fashion. The POR gene encodes P450 oxidoreductase, which transfers electrons to microsomal enzymes including three steroidogenic enzymes: P450c17 (17 $\alpha$-hydroxylase/17,20 lyase), P450c21 (21-hydroxylase), and P450aro (aromatase) [4]. In addition to impaired sexual development and steroidogenesis, POR-deficient patients exhibit skeletal malformations that likely occur because of deranged cholesterol biosynthesis during bone formation [5].

To date, only one confirmed ABS patient with a mutation in $P O R$ has been reported in Korea by molecular genetic methods [6]. Here, we report two cases of ABS with different clinical manifestations and disease progression, which were caused by mutations in the FGFR2 or POR genes.

Case

\section{Patient 1}

A female baby born at 34 weeks and 4 days of gestation with a birth weight of $2.91 \mathrm{~kg}$ was transferred to the neonatal intensive care unit of the Seoul National University Children's Hospital because of a respiratory difficulty at day 1 after birth. She cried well initially, but gradually developed a respiratory difficulty. She was the second baby of healthy non-consanguineous parents and her elder sister was healthy. She had facial dysmorphisms suggesting syndromic craniosynostosis, for example, proptosis, frontal bossing, and small anterior fontanelle. Venous blood gas analysis yielded the following data at arrival: $\mathrm{pH} 7.09$, $\mathrm{pCO}_{2} 90.4 \mathrm{mmHg}, \mathrm{pO}_{2} 47 \mathrm{mmHg}, \mathrm{HCO}_{3}{ }^{-} 27.4 \mathrm{mEq} / \mathrm{L}$. Therefore, intubation was performed. Because she also had a large and floppy epiglottis, several extubation attempts failed and she underwent tracheostomy at the age of 30 days. Multiple skeletal abnormalities were also evident. Craniofacial bone computed tomography (CT) scan revealed premature closure of the bilateral coronal sutures. Mid-facial hypoplasia caused proptosis (Fig. 1A). Both of elbow joints were fixed at about $100^{\circ}$ and radiohumeral synostosis was identified on X-ray findings. Thumbs and toes were broad and a skeletal survey showed a dorsally inverted coccyx (Fig. 1B). An anal-fissure-like skin defect on the perineum was observed. However, external genitalia were normal. Small patent ductus arteriosus was observed at day 1 after birth and no congenital anomalies of abdominal organs were noted. Newborn screening for inborn errors of metabolism showed a

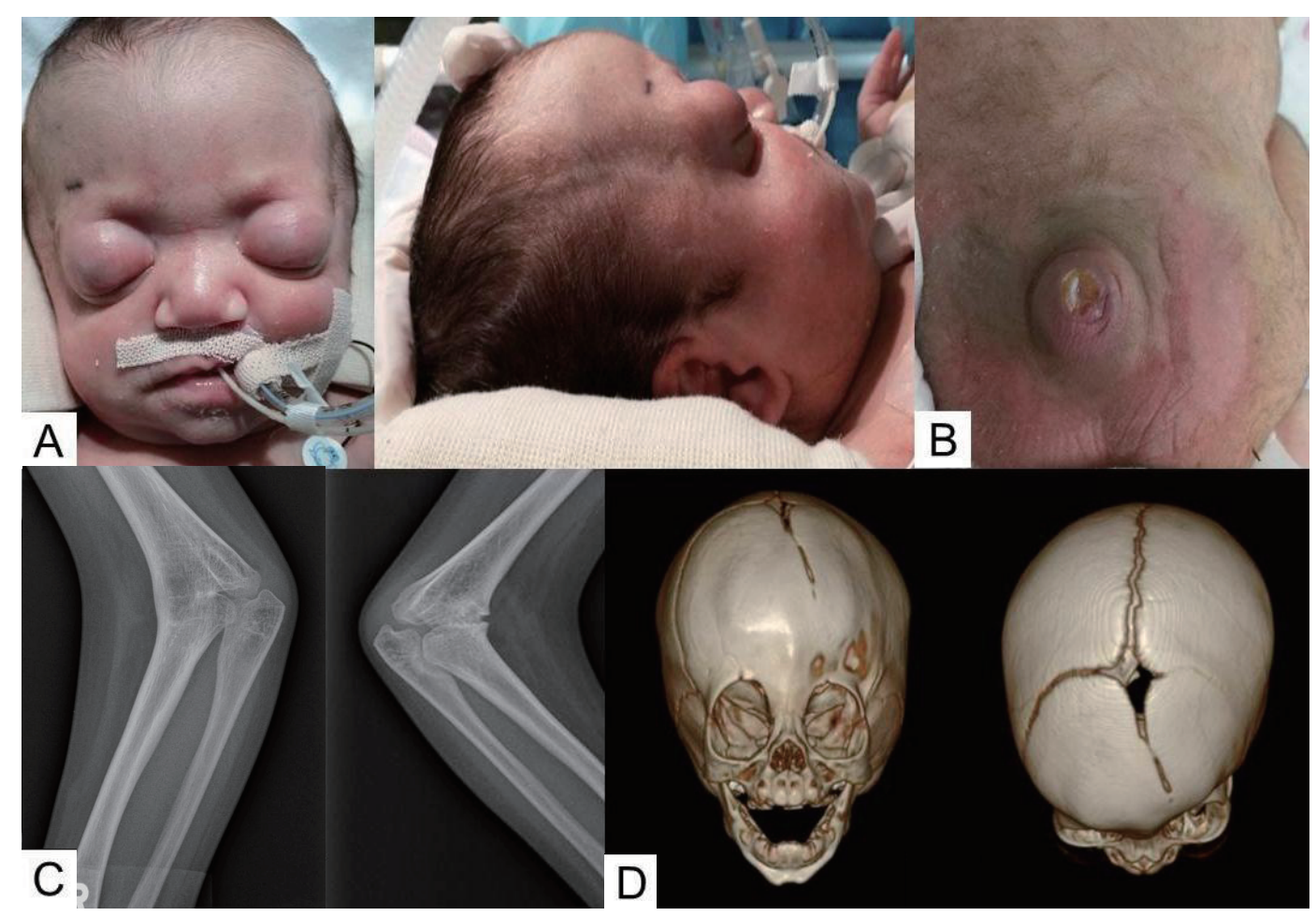

Fig. 1. Skeletal malformations in ABS patients. Facial photographs of Patient 1 show proptosis, mid-face hypoplasia with depressed nasal bridge (A) and dorsally inverted coccyx (B). In Patient 2, bilateral radio-humero-ulnar synostosis was demonstrated in simple radiographs at the age of 3 years $(C)$ and premature fusion of the left coronal and metopic sutures was found by computed tomography scanning (D). 
normal level of $17 \alpha$-hydroxyprogesterone (17-OHP). Standarddose adrenocorticotropic hormone stimulation test revealed normal adrenal steroidogenesis. She was confirmed as having ABS with the p.Tyr290Cys mutation by the FGFR2 gene analysis. No POR mutation was identified. At the age of 3 months, she underwent suturectomy of the bilateral coronal sutures and sagittal suture.

\section{Patient 2}

A male baby was born at 37 weeks and one day of gestation with a birth weight of $2.7 \mathrm{~kg}$ at a local obstetric hospital. He was the firstborn child of healthy non-consanguineous parents. At day 14 after birth, he visited the outpatient clinic at the Seoul National University Children's Hospital due to progressive respiratory difficulty. Glossoptosis and choanal atresia were identified. He also showed a syndromic face with proptosis, micrognathia, and low-set, deformed ears. He had bilateral cryptorchidism and limitations of joint movement in both elbows. Simple radiographs showed bilateral humero-radioulnar synostosis (Fig. 1C). There were no congenital anomalies of the heart and abdominal organs. On the newborn screening test of inborn errors of metabolism, his 17-OHP level was found to be elevated (17-OHP $18.2 \mathrm{ng} / \mathrm{mL}$, reference range $<7.0 \mathrm{ng} /$ $\mathrm{mL}$ ) and congenital adrenal hyperplasia was diagnosed by the adrenocorticotropic hormone stimulation test (Table 1). Therefore, treatment with hydrocortisone and fludrocortisone was started at the age of 50 days, and subsequently, hyponatremia and hyperkalemia were corrected so that sodium and potassium concentrations returned to normal ranges. At the age of 6 months, early closure of the anterior fontanelle was observed and premature fusion of the left coronal and metopic sutures was revealed on craniofacial CT findings (Fig. 1D). Therefore, the patient underwent cranioplasty. Arthrolysis for bilateral radio-humeral synostosis was performed at the age of 3 years. During the follow-up, he also showed symphalangism of the second to fifth metacarpal phalangeal joints and bony fusions between the capitate and hamate bones. ABS was confirmed by the $P O R$ gene analysis and two previously reported mutations, p.Arg457His and p.GIn555Profs*19, were identified (Fig. 2). His parents were heterozygous carriers of each POR mutation. There was no mutation in the FGFR2 gene. The patient is 7 years old at present. He consistently takes hydrocortisone and fludrocortisone and undergoes regular check-ups to monitor serum 17-0HP, cortisol, and renin levels.

Table 1. Results of the adrenocorticotropic hormone (ACTH) stimulation test in Patient 2

\begin{tabular}{|c|c|c|c|c|c|c|}
\hline & \multicolumn{6}{|c|}{ Age (mo) } \\
\hline & \multicolumn{2}{|c|}{1} & \multicolumn{4}{|c|}{14} \\
\hline ACTH dose $(\mu \mathrm{g})$ & \multicolumn{2}{|c|}{125} & \multicolumn{2}{|c|}{250} & \multicolumn{2}{|c|}{1} \\
\hline Test results & Baseline & $60 \mathrm{~min}$ & Baseline & $120 \mathrm{~min}$ & Baseline & $60 \mathrm{~min}$ \\
\hline ACTH (pg/mL) & 146 & & 315 & & 315 & \\
\hline 17-OHP (ng/dL) & 85.0 & 98.0 & 21.0 & 58.0 & 21.0 & 55.0 \\
\hline Cortisol ( $\mu \mathrm{g} / \mathrm{dL})$ & 18.1 & 16.9 & 11.0 & 9.1 & 11.0 & 11.2 \\
\hline 17-0HP/cortisol ratio & 4.69 & 5.79 & 1.90 & 6.37 & 1.90 & 4.91 \\
\hline
\end{tabular}

Reference ranges: ACTH, 7-28 pg/mL; 17-OHP, 11-170 ng/dL; cortisol, 3-23 $\mu \mathrm{g} / \mathrm{dL}$.

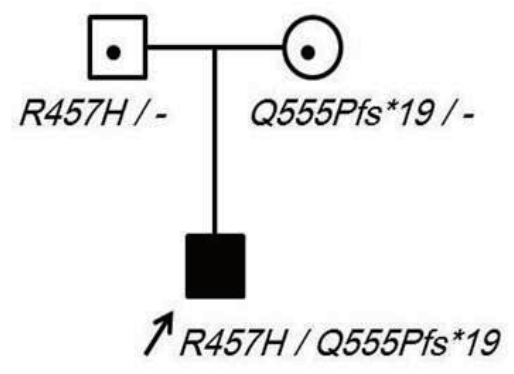

5 C A G G C C CR R T A C TACT

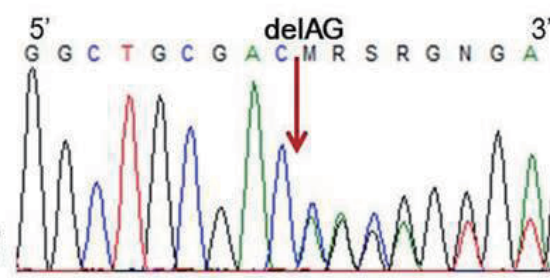

Fig. 2. Partial genomic DNA sequence of the $P O R$ gene of patient 2. Patient 2 had the following compound heterozygote mutations: the missense mutation p.Arg457His and the frame-shift mutation p.Gln555Profs*19. Both of the patient's parents are heterozygous carriers of each mutation. 
Neuropsychological development is appropriate for his age with a full-scale intelligence quotient of 115 .

\section{Discussion}

The most characteristic feature of ABS is craniosynostosis, which often leads to mid-facial hypoplasia and skeletal malformations including systemic bony fusions, especially radiohumeral or radioulnar synostosis. $A B S$ is one of the craniosynostosis syndromes related to mutations in the FGFR2 gene. The clinical manifestations of ABS were first described by Antley and Bixler [7] in 1975, and an FGFR2 mutation was discovered to be the cause of ABS in 1998 [8]. In 2004, it was revealed that mutations in another gene, $P O R$, are associated with ABS [3]. POR mutations cause not only the skeletal phenotypes of $A B S$ but also congenital adrenal hyperplasia. Gain-of-function mutations in FGFR2 and lossof-function mutations in $P O R$ result in identical skeletal and other dysmorphic features $[9,10]$. The $P O R$ gene encodes a key enzyme for transferring electrons to microsomal P450 enzymes, which is the rate-limiting step enzyme in steroid biosynthesis. The mechanism of skeletal malformations in ABS with POR mutations is not completely understood. However, some evidence suggests that disturbances of cholesterol biosynthesis may induce skeletal malformations. Smith-LemliOpitz syndrome is a well-known example of having a skeletal malformation caused by cholesterol biosynthesis impairment [11]. POR mutations inhibit the activity of several enzymes involved in cholesterol biosynthesis, such as CYP51A1, squalene monooxygenase, CYP26A1, CYP26B1, and CYP26C1 [12]. Furthermore, $A B S$ can be triggered iatrogenically by fluconazole, which acts by inhibiting CYP51 activity during the first trimester of pregnancy [13]. Therefore, further studies should investigate the involvement of each step of the cholesterol synthesis pathway in skeletal development and determine those steps, whose deregulation is directly responsible for the skeletal features of $\mathrm{ABS}$ due to $P O R$ mutations. Furthermore, the relationship between the FGFR2 and $P O R$ gene products remains to be fully elucidated.

In our patient with POR mutations (Patient 2), multiple joint synostosis tended to progress with age in contrast to the case of the patient with mutated FGFR2 (Patient 1). Craniosynostosis was detected after proptosis progressed from the age of 6 months. Additionally, other joint contractures, including humero-radio-ulnar synostosis, were evident during the followup.
Although ABS can initially be suspected on the basis of craniofacial manifestations and radiological findings of skeletal malformations, early identification of the genetic cause is helpful to establish a long-term treatment plan for accompanying complications, such as congenital adrenal hyperplasia, and to provide appropriate genetic counseling for patients and their families.

In conclusion, we reported two cases of ABS caused by mutations in the FGFR2 and POR genes. ABS patients require multiple operations for osteolysis on skull sutures and elbow joints. Moreover, all ABS patients with POR mutations should be treated with steroid hormones in stress conditions, such as operation and severe infection. Therefore, we recommend an early ACTH stimulation test and prompt molecular genetic testing for mutations in the FGFR2 and POR genes in all patients suspected to have ABS.

\section{References}

1. Senarath-Yapa K, Chung MT, McArdle A, Wong VW, Quarto N, Longaker MT, et al. Craniosynostosis: molecular pathways and future pharmacologic therapy. Organogenesis 2012;8:103-13.

2. Agochukwu NB, Solomon BD, Muenke M. Impact of genetics on the diagnosis and clinical management of syndromic craniosynostoses. Childs Nerv Syst 2012;28:1447-63.

3. Miller WL. P450 oxidoreductase deficiency: a new disorder of steroidogenesis with multiple clinical manifestations. Trends Endocrinol Metab 2004;15:311-5.

4. Sahakitrungruang $T$, Huang $N$, Tee MK, Agrawal V, Russell WE, Crock $\mathrm{P}$, et al. Clinical, genetic, and enzymatic characterization of P450 oxidoreductase deficiency in four patients. J Clin Endocrinol Metab 2009:94:4992-5000.

5. Flück CE, Miller WL. P450 oxidoreductase deficiency: a new form of congenital adrenal hyperplasia. Curr Opin Pediatr 2006;18:435-41.

6. Ko JM, Cheon CK, Kim GH, Yoo HW. A case of Antley-Bixler syndrome caused by compound heterozygous mutations of the cytochrome P450 oxidoreductase gene. Eur J Pediatr 2009;168:877-80.

7. Antley R, Bixler D. Trapezoidocephaly, midfacial hypoplasia and cartilage abnormalities with multiple synostoses and skeletal fractures. Birth Defects Orig Artic Ser 1975;11:397-401.

8. Chun K, Siegel-Bartelt J, Chitayat D, Phillips J, Ray PN. FGFR2 mutation associated with clinical manifestations consistent with Antley-Bixler syndrome. Am J Med Genet 1998;77:219-24.

9. Huang N, Pandey AV, Agrawal V, Reardon W, Lapunzina PD, Mowat D, et al. Diversity and function of mutations in p450 oxidoreductase in patients with Antley-Bixler syndrome and disordered steroidogenesis. 
Am J Hum Genet 2005;76:729-49.

10. Miller WL. P450 oxidoreductase deficiency: a disorder of steroidogenesis with multiple clinical manifestations. Sci Signal 2012;5:pt11.

11. Wassif CA, Maslen C, Kachilele-Linjewile S, Lin D, Linck LM, Connor $W E_{1}$ et al. Mutations in the human sterol delta7-reductase gene at 11q12-13 cause Smith-Lemli-Opitz syndrome. Am J Hum Genet
1998;63:55-62.

12. Fukami M, Ogata T. Cytochrome P450 oxidoreductase deficiency: rare congenital disorder leading to skeletal malformations and steroidogenic defects. Pediatr Int 2014;56:805-8.

13. Reardon W, Smith A, Honour JW, Hindmarsh P, Das D, Rumsby G, et al. Evidence for digenic inheritance in some cases of Antley-Bixler syndrome? J Med Genet 2000;37:26-32. 